\title{
Image fusion techniques in permanent seed implantation
}

\author{
Alfredo Polo, MD, PhD \\ Brachytherapy and Intraoperative Radiotherapy Unit, Radiation Oncology Department, Ramon y Cajal University Hospital, Madrid, Spain
}

\begin{abstract}
Over the last twenty years major software and hardware developments in brachytherapy treatment planning, intraoperative navigation and dose delivery have been made. Image-guided brachytherapy has emerged as the ultimate conformal radiation therapy, allowing precise dose deposition on small volumes under direct image visualization. In this process imaging plays a central role and novel imaging techniques are being developed (PET, MRI-MRS and power Doppler US imaging are among them), creating a new paradigm (dose-guided brachytherapy), where imaging is used to map the exact coordinates of the tumour cells, and to guide applicator insertion to the correct position. Each of these modalities has limitations providing all of the physical and geometric information required for the brachytherapy workflow. Therefore, image fusion can be used as a solution in order to take full advantage of the information from each modality in treatment planning, intraoperative navigation, dose delivery, verification and follow-up of interstitial irradiation. Image fusion, understood as the visualization of any morphological volume (i.e. US, CT, MRI) together with an additional second morphological volume (i.e. CT, MRI) or functional dataset (functional MRI, SPECT, PET), is a well known method for treatment planning, verification and follow-up of interstitial irradiation. The term image fusion is used when multiple patient image datasets are registered and overlaid or merged to provide additional information. Fused images may be created from multiple images from the same imaging modality taken at different moments (multi-temporal approach), or by combining information from multiple modalities. Quality means that the fused images should provide additional information to the brachytherapy process (diagnosis and staging, treatment planning, intraoperative imaging, treatment delivery and follow-up) that cannot be obtained in other ways. In this review I will focus on the role of image fusion for permanent seed implantation.

J Contemp Brachyther 2010; 2, 3: 98-106 DOI: 10.5114/jcb.2010.16920
\end{abstract}

Key words: brachytherapy, image fusion, prostate cancer, seeds.

\section{Purpose}

Modern brachytherapy relies on modern imaging techniques. With the advent, twenty years ago, of CT-based dose planning a more detailed knowledge of the dosevolume relationship in brachytherapy was possible. However, this knowledge was only partial due to the poor resolution of CT for target volume delineation when applicators are in place and the lack of temporal information of organ motion, very important in brachytherapy due to the marked gradients involved in dose delivery.

In prostate seed implantation, transrectal ultrasound (TRUS) provides adequate imaging of the soft tissue anatomy but it does not allow for robust localization of the implanted vectors. Various researchers have tried to use TRUS to segment the seeds in permanent prostate brachytherapy, with varying results [1-3].

Magnetic resonance imaging (MRI) can overcome some of the limitations inherent in CT and US. MRI offers a threedimensional (3D) dataset, arbitrary multiplanar reconstruction and better soft-tissue resolution with good correlation with TRUS-based evaluations and pathological findings, making it an attractive image modality for brachytherapy dosimetry [4-11]. Various experiences using MRI for permanent seed implantation have been reported [12,13]. On the other hand, recent technological advances in radiology and nuclear medicine gave us more understanding of the topography and metabolism of tumours. Functional imaging will open a new dimension to optimize radiation therapy, including brachytherapy [14]. Image fusion, understood as the visualization of any morphological volume (i.e. US, CT, MRI) together with an additional second morphological volume (i.e. CT, MRI) or functional dataset (functional MRI, SPECT, PET), is a wellknown method for treatment planning, verification and follow-up of interstitial irradiation. In this review I will focus on the role of image fusion for permanent seed implantation.

\section{Image fusion in brachytherapy}

Medical imaging has gained influence and is nowadays a fundamental tool in brachytherapy. Almost every aspect 
of patient management in brachytherapy involves multimodality imaging. Imaging is used for diagnosis and staging, for treatment planning, for intraoperative navigation, treatment delivery and for monitoring patients after brachytherapy. Whereas computed tomography (CT) is the primary modality for treatment planning in brachytherapy, other modalities such as magnetic resonance imaging (MRI), either morphological or functional, positron and single photon emission computed tomography (PET and SPECT) and ultrasound imaging (US) are often used in combination for treatment planning refinement $[15,16]$. On other occasions, those image modalities can be used intraoperatively for image guidance [17]. Magnetic resonance imaging provides excellent soft tissue contrast, allowing superior delineation of normal tissues and tumour volumes [18]. Functional MRI, SPECT and PET provide unique metabolic information capable of resolving ambiguities in anatomical image data and can quantify partial organ function [19]. Ultrasound imaging provides real-time volumetric information for delineating organ boundaries for both treatment planning and treatment delivery, and is the base image for prostatic brachytherapy. New developments in ultrasound imaging such as elastography or power Doppler open a door to intraoperative functional imaging [20].

The data from all these modalities help the radiation oncologist to develop a more complete description of the patient's disease. Each of these modalities alone, however, does not provide all of the physical and geometric information required for brachytherapy planning. Therefore, in order to take full advantage of the information from each modality, image fusion is a well-known method for treatment planning, verification and follow-up of interstitial irradiation [21].

The term image fusion is used when multiple patient image datasets are registered and overlaid or merged to provide additional information [22]. Fused images may be created from multiple images from the same imaging modality taken at different moments (multi-temporal approach), or by combining information from multiple modalities. The goal of image fusion is to integrate complementary information from different patient datasets into one new image containing information the quality of which cannot be achieved otherwise. Quality means, in this context, that the fused images should provide additional information for the brachytherapy process (diagnosis and staging, treatment planning, intraoperative imaging, treatment delivery and follow-up) that cannot be obtained in other ways.

\section{General technique for image fusion}

The process of image fusion involves two main tasks. The first task is to estimate the parameters of the coordinate transformation that relates homologous points in the two studies (dataset registration). The second task is to apply the resultant transformation to map structures or features of interest from one study to another (structure mapping) or to directly combine greyscale data from the two studies (image fusion) [21].

\section{Dataset registration}

This is the process to estimate the coordinate transformation relating homologous points in two imaging studies. The general approach is to plan a method of measuring the degree of mismatch between homologous features in two datasets and to use standard numerical methods to determine the transformation parameters that minimize (maximize) this value. Attributes used to compute this value are typically geometric structures (homologous points, lines or surfaces or combinations of these) extracted from the datasets or the native greyscale data. The situation is more difficult when the anatomy involved is not rigid (non-rigid dataset registration). In these cases, a more complicated transformation involving a larger number of degrees of freedom is required for registering the data adequately. Organ deformation due to applicator placement or the use of invasive imaging modalities (transrectal probes for ultrasound or magnetic resonance imaging) makes non-rigid registration needed for planning image fusion protocols for brachytherapy [23].

\section{Structure mapping and image fusion}

Once the transformation relating imaging studies is calculated, data relating to one study may be integrated or fused with those of another. There are two methods to achieve this integration. The first method, called structure mapping, involves mapping the outlines for the volumes of interest from one study to the other. The second, called image fusion, involves transforming and reformating image data from one study to match the orientation and scale of another study, which allows for simultaneous visualization of greyscale information from corresponding anatomical planes.

\section{Quality assurance of image fusion}

The process of image fusion described above requires verification of the results. Quality assurance is indeed a critical point before routinely implementing image fusion in the patient workflow in radiation oncology [24].

The American Association of Physicists in Medicine (AAPM) Task Group 53 Report outlined general commissioning and routine procedural quality assurance (QA) checks to be used for treatment planning [25]. They also stated that multimodality image registration is a complex area and required further development and its own task group. A task group (TG 132) was created by the AAPM to review the techniques for image registration, to identify issues related to clinical implementation, to determine the best methods to assess accuracy, and to outline issues related to acceptance and QA.

Image fusion quality assurance requires objective metrics for image registration evaluation. Phantom testing determines algorithm variations and calibration limits. However, phantoms do not completely capture other factors affecting image registration algorithms, such as variations in slice thickness, resolution, distortion, noise, and patient movement [26-28]. Setup variations and irregularity of patients are limiting factors that can be reduced by cross-comparison of redundant structures. When objective 
metrics are not calculable, quality assurance can be addressed through qualitative assessment. This can include image overlay and side-by-side comparison.

In conclusion, phantom-based quality assurance can confirm performance of imaging devices, registration software, and network storage and retrieval, but visual checks ensure consistency between patient cases. This requires logical, sequential, and reproducible processes to eliminate the possibility of human error.

\section{Image fusion for post-implant dosimetry}

Control of the delivered dose with seed implantation has been more elusive than that with external beam radiotherapy (EBRT), because of its uncertainties [29-33]. Postimplant dosimetry is a standard tool for assessing implant quality. Currently, both the ABS (American Brachytherapy Society) and the AAPM (American Association of Physicists in Medicine) recommend the execution of postimplant dosimetric analysis for all patients undergoing permanent seed implantation [31-34]. The post-implant dosimetry permits the documentation of the actual dose delivered to the prostate and the OAR. Secondly, the calculated dosimetric parameters create a "learning curve" and help to refine the technique. Data obtained from postimplant dosimetry can be used for future clinical outcome analysis. Finally, post-implant dosimetry allows the comparison of clinical datasets from various institutions, and consequently a quality control tool for multicentric clinical trials [35].

At present, CT-based post-implant analysis is the most commonly used method for carrying out quantitative dosimetric evaluations. Stock et al. [33] found a dose-response relationship for I-125 prostate implants using CT-based dosimetry, identifying a cut-off value of 140 Gy predicting prostate-specific antigen (PSA) relapse-free survival. In a recent comprehensive review of CT-based dosimetry parameters and biochemical control including 790 patients treated with permanent seed implantation, Potters et al. [32] found a cut-off value of $90 \%$ of the prescribed dose predicting PSA relapse-free survival. Wallner et al. [36] also showed that CT-based dosimetry can predict which patients are at higher risk of radiation-related morbidity.

However, the determination of the dose delivered to the target and to the OAR is highly dependent on how accurately the target volume is defined in the CT image set [34], making accurate and reproducible delineation a key point when evaluating seed implant quality by means of dosevolume histogram (DVH) analysis. Although Badiozamani et al. [37] reported high consistency between TRUS and CT-based prostate volumes, several studies have observed discrepancies in prostate volume, as outlined by TRUS, MRI and CT, showing difficulties in differentiating the prostate gland from periprostatic tissues when using CT [31,38-40]. Several studies have reported significant differences in inter- and intra-observer accuracy in outlining the prostate on CT images [41-46]. Due to the very small difference in density, the prostate gland is not well resolved from other adjacent soft tissue structures, usually resulting in prostate volume overestimation with respect to the
TRUS-based volume used to plan the implant. Critical prostate interfaces to outline in CT images are: the posterior portion of the prostate/anterior wall of the rectum, the postero-apical prostate/anterior portion of levator ani muscles and neurovascular bundles/prostate gland [38,39,47]. Narayana et al. [38] using two different approaches for preplanning seed implantation (CT vs. TRUS-based) found a significant difference with CT volumes, markedly greater $(+47 \%)$ than TRUS volumes. For EBRT, Roach et al. [39] compared prostate volumes defined by MRI and CT in 20 patients. On average, the mean prostate volume was $32 \%$ larger for CT compared to MRI, corresponding to an average 8.7 c.c. larger prostate. Kagawa et al. [48] also reported a mean $26 \%$ overestimation when delineating prostate with CT images compared to MRI images. Rasch et al. [49] in their inter-observer study report CT-derived prostate volumes larger than MRI-derived volumes (median overestimation, $43 \%$ ), especially in the directions of the seminal vesicles and of the apex of the prostate. Merrick et al. [50] reported 25\% overestimation of prostateonly (without margins) volume when using CT-based calculations on day 0 post-implant. Lee et al. [51] reporting their experience in prostate seed implantation found a median CT overestimation of prostate gland volume compared to pre-implant TRUS of $31 \%$ for post-implant dosimetry being calculated 30 days after the implantation. In their centralized multicentric post-implant analysis, Bice et al. [52] reported a CT/TRUS ratio ranging between $2 \%$ and $74 \%$ (median value not available) with postimplant CT scans having been taken between day 1 and day 30 after the implantation.

MRI has a better soft-tissue resolution and MR-image based contours correlate better with TRUS-based evaluations and pathological findings, making it an attractive image modality for brachytherapy dosimetry [5,7-11]. Also a better inter-observer reproducibility has been described for MRI [43]. However, the signal in MRI-T2 weighted imaging is not so good to allow an easy and reliable identification of the seeds. Additionally, MR facilities are not usually available for radiotherapy treatment planning and its use is restricted to a few centres with limited cost-effectiveness. Several authors have reported their results with MRI-based dosimetry. Moerland et al. [53] analysed 21 patients undergoing I-125 seed implantation. Dosimetric calculations were made within the first three days after the implantation matching cross-sectional MRI images with isocentric radiographs for seed identification. The accuracy of the fusion process was acceptable. The DVH-based V100 was on average $60 \% \pm 10 \%$ [32 to $71 \%$ ]. Dubois et al. [54] report the first MRI-only-based post-implant results. Using an optimized MRI sequence that accentuated the artefact produced by the sources providing good target definition, they reported the results of a comparison of CT-based dosimetry with MRI-based dosimetry in 20 patients. They found a good agreement between the two techniques, with no statistical difference between volumes at the prescription isodose. They report no differences in either prostate volumes or in the DVHrelated parameters for the two image modalities used. Prete et al. [55] reported a comparison in dosimetric out- 
come for 15 patients undergoing seed implantation, using both CT and MRI-based dosimetry. There was an overestimation of prostate volume as determined from MRI of about 9\% larger than CT. DVH-related V100 was 78.6\% for the MRI group vs. $79 \%$ for the CT group ( $p=0.208)$. The surprising differences in volume from MRI to CT are attributed to the more subjective nature of visualizing the prostate contour on CT as opposed to MRI images, resulting in a smaller, and perhaps erroneous determination of CT volume.

To overcome the seed identification problems in MRIbased dosimetry, CT-MRI image fusion was first suggested by Amdur et al. [56] as a sophisticated way of assessing the quality of a prostate seed implant. Other authors have reported results using CT-MRI fusion protocols for postimplant dosimetry evaluation [48,49,53,56-64]. Polo et al. reported that CT/MRI fusion-based dosimetry allows accurate determination of the prostate size and significantly improves the dosimetric evaluation when compared with CT-based dosimetry [61]. McLaughlin et al. report that T2-weighted MR images provide superior prostate definition to T1-weighted images and T1-weighted fat saturation images [58].

\section{Image fusion techniques for dynamic dose calculation}

Dynamic dose calculation represents a paradigm shift in dose prescription and specification and source delivery for permanent seed implantation. It will mirror the imageguided radiation therapy (IGRT) paradigm in EBRT in that an intended prescription dose is adaptively "painted" to a changing 3D target volume. This process of 'dose painting' may result in alteration of a previously accepted isodose distribution and total implanted activity at any time, until the end of the procedure when a satisfactory dose delivery is achieved. In comparison to interactive planning, dynamic dose calculation relies on full 3D dose planning based on the real position of the delivered seeds. Instead of having interactive dose calculations based on a surrogate for the seed, dynamic dose planning will constantly update dose distribution using continuous deposited seed position feedback. It will account for seed migration, needle motion and misalignment and prostate gland changes in shape and volume [65].

Sometimes, the difference between interactive planning and dynamic dose calculation is difficult to address. Sometimes, what is called dynamic dose calculation by some authors is in fact interactive planning after a careful reading of the method described in the corresponding article. Dynamic dose calculation relies on continuous deposited seed feedback. It requires specific technology with the potential to constantly update calculations of dose distribution (dynamic dose calculation) as the implant proceeds. It is essential that the exact seed position is known in three dimensions. Improvements in imaging are thus required, as TRUS image degradation with time is a major issue that impairs seed visualization. The advantage of dynamic dose calculation is its potential to adjust the treatment plan to account for intra-prostatic seed migration as each seed is deposited and for the potential displacements of dropped seeds, caused by changes in the prostate position and volume during the implant procedure from oedema or trauma. Currently it is not commercially available. It is not yet possible to track individual deposited seeds reliably, and have this information imported back into the treatment planning system in real time. Methods have been described that register dropped seeds with ultrasonography or fluoroscopy, with adjustments to treatment plans made accordingly [66].

Several workflows have been outlined for dynamic dose calculation $[67,68]$. Lee et al. [68] describe the intraoperative dynamic dose optimization (IDDO) algorithm. The general scheme for performing IDDO consists of three steps: first, at some point during the implant, coordinates of implanted seeds are identified. Second, seed images are projected onto the reference frame of the ultrasound images for planning; and finally the plan is reoptimized. An optimal treatment plan is first obtained based on initial operating room-acquired ultrasound images. The intraoperative dosimetric evaluation is based on the fusion between ultrasound images and 3D seed coordinates reconstructed from fluoroscopic projections. With real-time treatment planning, it is possible to dynamically reoptimize treatment plans to account for actual seed positions (as opposed to planned positions) and needle-induced swelling to the gland during implantation. Postimplant analysis showed that the final seed configuration resulting from the IDDO method yields improved dosimetry (complete coverage and good conformity, thus sparing excess radiation to critical tissues).

Intraoperative dosimetric quality assurance in prostate brachytherapy (dynamic dose calculation) critically depends on discerning the 3D locations of implanted seeds. The ability to reconstruct the implanted seeds intraoperatively will allow us to make immediate provisions for dosimetric deviations from the optimal implant plan. However, the current technology needs further development in order to track seeds reliably. Currently available possibilities for seed tracking include: pure intraoperative TRUS-based tracking, TRUS-fluoroscopy fusion and intraoperative cone-beam CT.

\section{Dynamic dose calculation based on TRUS-fluoroscopy fusion}

Fluoroscopy was first used as a solo guidance modality for brachytherapy and other minimally invasive percutaneous procedures [1]. Mobile C-arms are ubiquitous in contemporary prostate brachytherapy and C-arm fluoroscopy is considered as the gold standard for intraoperative visualization of brachytherapy seeds. Some authors have described the combined use of TRUS and fluoroscopy, to create a reliable method for intraoperative seed capture and dosimetry optimization. Combining their images by spatial co-registration (fusion) offers the potential for a practical intraoperative dosimetric assessment. TRUS offers the ability to identify the prostate gland, and fluoroscopy can provide the data needed to perform 3D seed reconstruction. However, TRUS-fluoro intraoperative dosimetric analysis is yet to become a standard of care 
across hospitals. This is in part because of the workload involved in this procedure. On the other hand, digital $\mathrm{C}$-arms with encoded rotational joints are expensive machines that are not widely available. Finally, there are some unresolved technical problems: first, C-arm distortion correction and calibration; second, C-arm pose tracking; third, seed segmentation; fourth, seed matching and reconstruction; and finally, registration of C-arm to TRUS images.

Su et al. $[69,70]$ describe a fluoroscopic to TRUS fusionbased approach for intraoperative prostate brachytherapy dosimetry. Seed images were identified from multiple fluoroscopic images and reconstructed to determine the 3D distribution. Seeds identified from the TRUS images were used as fiducials for the registration between fluoroscopic and TRUS images. Less than $0.2 \%$ error in the D90 value was observed using the TRUS-fluoroscopy imagefusion-based method relative to the CT-based post-implantation dosimetry. However, in the phantom study, an average distance of $3 \mathrm{~mm}$ was observed between the seeds identified from TRUS and the reconstructed seeds at registration. This registration error can be improved by using more sophisticated registration algorithms that have outlier rejection capabilities, since artefacts in the TRUS images can be mistaken as seeds. Due to the fact that the correspondence of seeds between TRUS images and fluoroscopic images cannot be easily established, the number of seeds identified from TRUS should be large enough to represent the seed distribution for meaningful registration. Considerable human interaction is needed for the seed image identification process to remove false positives in the segmentation results. False positive seed images identified from the fluoroscopic images will cause false positives in the reconstructed 3D seeds. Although the seed reconstruction algorithm can compensate for the undetected seeds due to overlapping seed images, it does not have the capability of eliminating false positives. Another source of false positives is caused by the action of the seed reconstruction algorithm whereby a single seed image may be reused more than once. A more advanced technique is needed to reduce the likelihood of generating false positives and detect all the implanted seeds.

French et al. [71] present a new approach for intraoperatively computing dosimetry for prostate brachytherapy. The approach uses transrectal ultrasound (TRUS) and fluoroscopic images. The radiation oncologist manually registers the intraoperative TRUS images with the TRUS images from the preoperative volume study. Next, the fluoroscopic and TRUS images are registered using a single fluoroscopic image of the TRUS probe. The C-arm is not moved during the procedure and all images are acquired from the same $\mathrm{C}$-arm angles. The needles are then inserted using the needle guide and using visual feedback from TRUS. Before the needle is retracted, the needle tip is located in the TRUS image, and, using the known entry point of the needle in the needle guide, a needle path is interpolated. Fluoroscopic images of the patient's coronal plane are acquired throughout the procedure. After the acquisition of each fluoroscopic image, the $(x, z)$ coordinates of the implanted seeds are located in the image and back-projected to the TRUS image frame. Then, the y-coordinate of each seed is computed using the $(x, z)$ coordinates of the seeds and the interpolated needle path. The coordinates of the seeds are used to compute and display dosimetry with respect to the TRUS images. This allows the radiation oncologist to modify the preoperative plan to reflect the actual location of the implanted seeds. The radiation oncologist returns to inserting needles, repeating the above-described steps until all the needles have been inserted. The authors present clinical results of 5 patients implanted with the dynamic dose calculation described above. Values for D90 were very high (mean $201 \mathrm{~Gy}$, range 143.3-265.4 Gy) and target coverage ranged from 85.5 to $100 \%$ with a mean value of $93.1 \%$. For more accurate results, the authors issued intraoperative seed motion tracking and intraoperative oedema and present a method to achieve such tracking.

Gong et al. [72] investigate the feasibility of performing intraoperative dosimetry for prostate brachytherapy by fusing transrectal ultrasound (TRUS) and fluoroscopic data. Prostate phantoms were implanted with seeds, and four needles were inserted to create a reference system. The coordinates of these needles were determined in the TRUS frame of reference. The 3D needle tip positions were also calculated in the fluoroscopic coordinate system using a seed reconstruction method. The transformation between the TRUS and fluoroscopy coordinate systems was established by the least-squares solution using the singular value decomposition. The mean fiducial registration error was $0.8 \mathrm{~mm}$ and the test target registration error was $1.1 \mathrm{~mm}$. Alternatively, the comparison between the proposed method and CT-based dosimetry yielded a percentage of prostate volume receiving $100 \%$ and $150 \%$ of the prescribed minimal peripheral dose and minimal dose received by $90 \%$ of the prostate gland that agreed within $0.4 \%, 2.7 \%$, and $4.2 \%$, respectively. Using the proposed algorithm, the authors conclude that fusion between TRUS and fluoroscopic images can be used for intraoperative seed update and dosimetry optimization.

Jain et al. [1] explore the MARSHAL approach for the matching between 3D clouds of seeds and tracked ultrasound images. The authors propose a method for seed reconstruction from segmented C-arm fluoroscopy images. Using three presegmented images, matching and reconstruction of brachytherapy seeds using the Hungarian algorithm achieved complete matching in simulation experiments, and $98.5 \%$ in phantom experiments. Threedimensional reconstruction error for correctly matched seeds has a mean of $0.63 \mathrm{~mm}$, and $0.9 \mathrm{~mm}$ for incorrectly matched seeds. The maximum seed reconstruction error in each implant was typically around $1.32 \mathrm{~mm}$. Both on synthetic data and in phantom experiments, matching rate and reconstruction error achieved using presegmented images were found to be sufficient for prostate brachytherapy.

The underlying assumption of the previously reported papers has been that the coordinates for all the seeds are known, allowing intraoperative dosimetric update. However, segmentation algorithms are accurate segmenting seeds that are fully visible, leaving some other seeds unidentified, due to overlapping or poor ultrasound imaging. Thus the coordinates of unidentified seeds will not be available, resulting in some degree of dosimetric uncertainty [73]. This is an extremely important issue with 
clinical relevance. While some researchers have reported the number of hidden seeds to be between $7 \%$ and $45 \%$ of the total seeds, others have noticed only about $2-3 \%$ in their clinical data [1].

Combining intraoperative fluoroscopy with transrectal ultrasound is a very attractive modality for computing dynamic dosimetry for permanent seed implantation. However, it will not be viable unless it explicitly solves this problem. Solutions could arrive either from more precise segmenting algorithms, from improved ultrasound modalities, or from manufacturing novelties making seeds more eco-visible.

\section{Dynamic dose calculation based on TRUS-CBCT fusion}

Flat-panel imager-based cone-beam computed tomography $(\mathrm{CBCT})$ is a strong candidate technology for intraoperative imaging in image-guided procedures such as brachytherapy $[74,75]$. The soft-tissue imaging performance and potential navigational utility have been investigated by several authors both in pre-clinical and in clinical situations. In image-guided permanent seed implantation, soft-tissue imaging performance and seed detection could satisfy the imaging and navigation requirements. The demonstrated soft-tissue visibility, excellent spatial resolution, low imaging dose, and convenient form factor make C-arm-based cone-beam CT a powerful new technology for image-guidance applications, including brachytherapy, vertebroplasty and neurosurgery.

Siewerdsen et al. [76] describe the development of a multimode C-arm fluoroscopy, tomosynthesis and cone-beam CT for image-guided interventions in a wide range of clinical situations (neurosurgery, orthopaedics, spine surgery and radiotherapy). The key feature of the system is the integration of imaging, navigation, registration and display. The system has been tested from pre-clinical models to patient protocols: resection of palpable breast lesions, thoracic surgery (resection of superficial lung nodules, interventional radiology (guidance and evaluation of stent placement) and brachytherapy (guidance and verification of radioactive seeds in the prostate). In each application, the C-arm platform and multi-mode imaging functionality could offer a potentially significant advance over existing image-guidance approaches. This system offers some advantages, including patient access due to the open geometry, low radiation doses (facilitating repeat intraoperative scanning), portability (allowing service to multiple operating rooms), and low cost (relative to CT or MRI).

In a small study with 20 patients, Westendorp et al. [77] studied the feasibility of C-arm cone-beam computed tomography based postplanning and subsequent adaptation of underdosed critical areas by adding sources during permanent seed implantation. The proposed workflow was as follows: after finishing the implant, a CT study was made using the cone-beam CT C-arm unit following removal of the TRUS probe from the rectum. The 3D dataset was sent to the treatment planning system. Seeds were segmented in the CT images. With the patient still on the treatment couch, the delineated TRUS study was registered on the CT with labelled fiducials. The registration was performed using a least-squares algorithm allowing the marked positions to translate and rotate as a rigid body. The implanted fiducial markers were used as reference positions to align the TRUS and CT datasets. After registration, the root mean square of the distance between the corresponding fiducials in the TRUS and CT dataset was $0.8 \mathrm{~mm}$ on average. After the registration and the identification of all source positions from the CT image set, DVH analysis was performed. If the dosimetry was deemed satisfactory, the patient was discharged. Identification of sites with coverage deficiency led to the adoption of a corrective plan and the placement of remedial seeds. An average of four seeds were needed in the reported series. The position of the simulated remedial sources was specified according to the ultrasound targeting grid coordinates, just as in the original standard procedure planned preoperatively. The patient was prepared for reimplantation. The remedial seeds were implanted after the correction plan and the reimplant procedure was completed. A final cone-beam CT C-arm was obtained. The TRUS scan made after the first implantation procedure was registered on the CT images obtained, using the same procedure as used after the first implantation, resulting in final Day 0 dosimetry for the corrected implants. The entire procedure was scheduled to involve a time not exceeding $90 \mathrm{~min}$. Using this approach, the authors describe an improvement in the DVH-related parameters. For V100, the pre-corrected value was $83 \%$ of the target volume compared to $90 \%$ for the finished, corrected implant. For D90, the pre-corrected value was $95 \%$ of the prescription dose, rising to $100 \%$ once the patient was re-implanted. Finally, it has to be pointed out that the proposed workflow cannot be considered dynamic dose calculation, strictly speaking, because the seed tracking is dissociated from the implantation itself.

\section{Active research}

Active research is being done to bring dynamic dose calculation into the current clinical routine. However, some additional features are required for a full implementation. First, seed positions should be captured in real time. Second, the dosimetric update should be based on deposited seed location. Third, intraoperative prostate motion and shape and volume changes due to motion, swelling or bleeding should be accounted for. Finally, post-implant dosimetry is ideally performed at the end of the procedure, allowing final modifications before the patient exits the operating room.

\section{Functional imaging and permanent seed implantation}

Over the last few years, the use of molecular imaging has become increasingly popular in oncology, opening a new dimension to management for patients with cancer [78]. The potential role of functional imaging in radiation oncology can be broadly divided into four main areas [79]. First, functional imaging is emerging as a powerful technique in radiation treatment planning, assisting in target delineation [80]. Second, functional imaging would help 
in the modulation of the dose to the target volume (dose painting by numbers). Third, it can be used in assessing radiobiological processes during and after radiotherapy referred to as "radiodynamics". Finally, functional imaging can be utilized for in vivo predictive testing and in assessing the response to radiation therapy.

Recent advances now allow highly specific and sensitive detection of cellular and molecular events non-invasively. Theragnostic imaging for radiation oncology aims to map in three dimensions the distribution of a tumour, tissue, or functional feature, and to provide information about the clinical response of tumours or healthy tissues to radiotherapy [16]. In solid tumours, the aim is to provide images of phenotypic or microenvironmental characteristics known to affect the clinical response. Most research has been done to detect tumour burden and clonogen density, tumour hypoxia or proliferation. New markers will allow us to probe specific genetic pathways relevant to radiation therapy.

In the new paradigm (dose-guided brachytherapy), imaging is used to determine the coordinates of the tumour cells, and to guide applicator insertion to the correct position. To map cancer cells, a number of new imaging modalities have been developed in the last years: PET, MRI-MRS and power Doppler US imaging are among them. All those image modalities give twofold information: morphological on one side and metabolic on the other. Combining the two different aspects it is possible to define areas where it is likely that a tumour burden is present, or certain hypoxic areas, or areas of repopulation or intrinsic radiosensitivity load. Those areas are supposed to be liable to be boosted by high-precision modalities. In this setting, brachytherapy will offer the intrinsic advantages already mentioned. The rapid fall-off of the dose would serve to precisely sculpt the dose around these sub-volumes. This process is known as dose painting, as we can paint the different dose levels we want to achieve within the target volume. Correlation studies with pathological specimens are needed to check for spatial and temporal stability.

Imaging is also required for precise deposition of the prescribed dose. Beyond CT-based 3D planning and US needle guidance for prostate implantation there is a brand new field of "dose guidance" in which the brachytherapist can see in real time the relationship between the planned dose, the applicator and the anatomical volumes of interest. Different tools can be used (CT, MRI, US), each adapted to different clinical situations. Ultrasound is very suitable in the circumstances where brachytherapy is performed. It can be intraoperative, it is fast, there is no radiation exposure to the staff, it is cheap and it would allow direct visualization of the applicator and the intended dose overlaid together with anatomical and functional information. Fused images based on US could help the brachytherapist to precisely place the radioactive sources, according to the treatment plan.

\section{Conclusions}

Over the last twenty years major software and hardware developments in brachytherapy treatment planning, intra- operative navigation and dose delivery have been made. Image-guided brachytherapy has emerged as the ultimate conformal radiation therapy, allowing precise dose deposition on small volumes under direct image visualization. In this process imaging plays a central role and novel imaging techniques are being developed (PET, MRI-MRS and power Doppler US imaging are among them), creating a new paradigm (dose-guided brachytherapy), where imaging is used to map the exact coordinates of the tumour cells, and to guide applicator insertion to the correct position.

Each of these modalities has limitations in providing all of the physical and geometric information required for the brachytherapy workflow. Therefore, image fusion can be used as a solution in order to take full advantage of the information from each modality in treatment planning, intraoperative navigation, dose delivery, verification and follow-up of interstitial irradiation. Image fusion integrates complementary information from different patient datasets into a new one giving additional information relevant to the brachytherapy process.

The new paradigm in brachytherapy relies on the new image modalities for tumour mapping and dose guidance. Image fusion will be used in certain situations, as there is no "perfect" image modality for every step of the brachytherapy workflow. Brachytherapy will obtain a clear advantage from this modality that could translate into better treatments, more conformal to the target volume, more dose-intense, and less toxic to the surrounding tissues.

\section{References}

1. Jain AK, Zhou Y, Mustufa T et al. Matching and reconstruction of brachytherapy seeds using the Hungarian algorithm (MARSHAL). Med Phys 2005; 32: 3475-3492.

2. Han BH, Wallner K, Merrick G et al. Prostate brachytherapy seed identification on post-implant TRUS images. Med Phys 2003; 30: 898-900.

3. Xue J, Waterman F, Handler J et al. Localization of linked 125I seeds in postimplant TRUS images for prostate brachytherapy dosimetry. Int J Radiat Oncol Biol Phys 2005; 62: 912-919.

4. Penner EA. Interventional MR with a mid-field open system. In: Debatin JF (ed.). Interventional magnetic resonance imaging. Springer-Verlag, Berlin 1998; 11-18.

5. Hricak H, Jeffrey RB, Dooms GC et al. Evaluation of prostate size: a comparison of ultrasound and magnetic resonance imaging. Urol Radiol 1987; 9: 1-8.

6. Terris MK, Stamey TA. Determination of prostate volume by transrectal ultrasound. J Urol 1991; 145: 984-987.

7. Rahmouni A, Yang A, Tempany CM et al. Accuracy of in-vivo assessment of prostatic volume by MRI and transrectal ultrasonography. J Comput Assist Tomogr 1992; 16: 935-940.

8. Terris MK, McNeal JE, Stamey TA. Estimation of prostate cancer volume by transrectal ultrasound imaging. J Urol 1992; 147: 855-857.

9. al-Rimawi M, Griffiths DJ, Boake RC et al. Transrectal ultrasound versus magnetic resonance imaging in the estimation of prostatic volume. Br J Urol 1994; 74: 596-600.

10. Tewari A, Indudhara R, Shinohara K et al. Comparison of transrectal ultrasound prostatic volume estimation with magnetic resonance imaging volume estimation and surgical specimen weight in patients with benign prostatic hyperplasia. J Clin Ultrasound 1996; 24: 169-174.

11. Sosna J, Rofsky NM, Gaston SM et al. Determinations of prostate volume at 3-Tesla using an external phased array coil: comparison to pathologic specimens. Acad Radiol 2003; 10: 846-853. 
12. Cormack RA, Kooy H, Tempany CM et al. A clinical method for real-time dosimetric guidance of transperineal 125I prostate implants using interventional magnetic resonance imaging. Int J Radiat Oncol Biol Phys 2000; 46: 207-214.

13. Cormack RA, Tempany CM, D'Amico AV. Optimizing target coverage by dosimetric feedback during prostate brachytherapy. Int I Radiat Oncol Biol Phys 2000; 48: 1245-1249.

14. Polo A. Feasibility of functional imaging for brachytherapy. J Contemp Brachyth 2009; 1: 45-49.

15. Saw CB, Chen H, Beatty RE et al. Multimodality image fusion and planning and dose delivery for radiation therapy. Med Dosim 2008; 33: 149-155.

16. Bentzen SM. Theragnostic imaging for radiation oncology: dose-painting by numbers. Lancet Oncol 2005; 6: 112-117.

17. Polo A, Salembier C, Venselaar J et al. Review of intraoperative imaging and planning techniques in permanent seed prostate brachytherapy. Radiother Oncol 2010; 94: 12-23.

18. Villeirs GM, De Meerleer GO. Magnetic resonance imaging (MRI) anatomy of the prostate and application of MRI in radiotherapy planning. Eur J Radiol 2007; 63: 361-368.

19. Nimmagadda S, Ford EC, Wong JW et al. Targeted molecular imaging in oncology: focus on radiation therapy. Semin Radiat Oncol 2008; 18: 136-148.

20. Pallwein L, Mitterberger M, Pelzer A et al. Ultrasound of prostate cancer: recent advances. Eur Radiol 2008; 18: 707-715.

21. Image registration and data fusion in radiation therapy. 79 Spec No 1; 2006; The University of Michigan, Ann Arbor, MI 48103, USA 2006.

22. Image Fusion: Is It Merely the Blending of Pretty Pictures? 3(9); 2010; Cardiovascular Imaging Laboratory, Imaging Institute and Heart and Vascular Institute, Cleveland Clinic, Cleveland, Ohio 2010.

23. Christensen GE, Carlson B, Chao KS et al. Image-based dose planning of intracavitary brachytherapy: registration of serialimaging studies using deformable anatomic templates. Int J Radiat Oncol Biol Phys 2001; 51: 227-243.

24. Sharpe M, Brock KK. Quality assurance of serial 3D image registration, fusion, and segmentation. Int J Radiat Oncol Biol Phys 2008; 71: 33-37.

25. American Association of Physicists in Medicine Radiation Therapy Committee Task Group 53: quality assurance for clinical radiotherapy treatment planning. 25(10); 1998; University of Michigan Medical Center, Ann Arbor, USA. bfraass@umich.edu: 1998.

26. Phantom validation of coregistration of PET and CT for imageguided radiotherapy. 31(5); 2004; Department of Radiology and Radiological Sciences, Vanderbilt University, Nashville, Tennessee, USA.

27. Parker CC, Damyanovich A, Haycocks T et al. Magnetic resonance imaging in the radiation treatment planning of localized prostate cancer using intra-prostatic fiducial markers for computed tomography co-registration. Radiother Oncol 2003; 66: 217-224

28. Mutic S, Dempsey JF, Bosch WR et al. Multimodality image registration quality assurance for conformal three-dimensional treatment planning. Int J Radiat Oncol Biol Phys 2001; 51: 255-260.

29. Tanaka O, Hayashi S, Matsuo M et al. Comparison of MRIbased and CT/MRI fusion-based postimplant dosimetric analysis of prostate brachytherapy. Int J Radiat Oncol Biol Phys 2006; 66: 597-602.

30. Nag S, Beyer D, Friedland J et al. American Brachytherapy Society (ABS) recommendations for transperineal permanent brachytherapy of prostate cancer. Int J Radiat Oncol Biol Phys 1999; 44: 789-799.

31. Nag S, Bice W, DeWyngaert K et al. The American Brachytherapy Society recommendations for permanent prostate brachytherapy postimplant dosimetric analysis. Int J Radiat Oncol Biol Phys 2000; 46: 221-230.

32. Potters L, Cao Y, Calugaru E et al. A comprehensive review of CT-based dosimetry parameters and biochemical control in patients treated with permanent prostate brachytherapy. Int J Radiat Oncol Biol Phys 2001; 50: 605-614.

33. Stock RG, Stone NN, Tabert A et al. A dose-response study for I-125 prostate implants. Int J Radiat Oncol Biol Phys 1998; 41: 101-108.

34. Yu Y, Anderson LL, Li Z et al. Permanent prostate seed implant brachytherapy: report of the American Association of Physicists in Medicine Task Group No. 64. Med Phys 1999; 26: 2054-2076.

35. Bice WS, Jr., Prestidge BR, Prete JJ et al. Clinical impact of implementing the recommendations of AAPM Task Group 43 on permanent prostate brachytherapy using 125I. American Association of Physicists in Medicine. Int J Radiat Oncol Biol Phys 1998; 40: 1237-1241.

36. Wallner K, Roy J, Harrison L. Dosimetry guidelines to minimize urethral and rectal morbidity following transperineal I-125 prostate brachytherapy. Int J Radiat Oncol Biol Phys 1995; 32: 465-471.

37. Badiozamani KR, Wallner K, Cavanagh W et al. Comparability of CT-based and TRUS-based prostate volumes. Int J Radiat Oncol Biol Phys 1999; 43: 375-378.

38. Narayana V, Roberson PL, Winfield RJ et al. Impact of ultrasound and computed tomography prostate volume registration on evaluation of permanent prostate implants. Int J Radiat Oncol Biol Phys 1997; 39: 341-346.

39. Roach M 3rd, Faillace-Akazawa P, Malfatti C et al. Prostate volumes defined by magnetic resonance imaging and computerized tomographic scans for three-dimensional conformal radiotherapy. Int J Radiat Oncol Biol Phys 1996; 35: 1011-1018.

40. Rosen II, Khan KM, Lane RG et al. The effect of geometric errors in the reconstruction of iridium-192 seed implants. Med Phys 1982; 9: 220-223.

41. Al-Qaisieh B, Ash D, Bottomley DM et al. Impact of prostate volume evaluation by different observers on CT-based postimplant dosimetry. Radiother Oncol 2002; 62: 267-273.

42. Lee WR, Roach M 3rd, Michalski J et al. Interobserver variability leads to significant differences in quantifiers of prostate implant adequacy. Int J Radiat Oncol Biol Phys 2002; 54: 457-461.

43. Dubois DF, Prestidge BR, Hotchkiss LA et al. Intraobserver and interobserver variability of MR imaging- and CT-derived prostate volumes after transperineal interstitial permanent prostate brachytherapy. Radiology 1998; 207: 785-789.

44. Cazzaniga LF, Marinoni MA, Bossi A et al. Interphysician variability in defining the planning target volume in the irradiation of prostate and seminal vesicles. Radiother Oncol 1998; 47: 293-296.

45. Fiorino C, Reni M, Bolognesi A et al. Intra- and inter-observer variability in contouring prostate and seminal vesicles: implications for conformal treatment planning. Radiother Oncol 1998; 47: 285-292

46. Valicenti RK, Sweet JW, Hauck WW et al. Variation of clinical target volume definition in three-dimensional conformal radiation therapy for prostate cancer. Int J Radiat Oncol Biol Phys 1999; 44: 931-935.

47. Sandler HM, Bree RL, McLaughlin PW et al. Localization of the prostatic apex for radiation therapy using implanted markers. Int J Radiat Oncol Biol Phys 1993; 27: 915-919.

48. Kagawa K, Lee WR, Schultheiss TE et al. Initial clinical assessment of CT-MRI image fusion software in localization of the prostate for 3D conformal radiation therapy. Int J Radiat Oncol Biol Phys 1997; 38: 319-325.

49. Rasch C, Barillot I, Remeijer P et al. Definition of the prostate in CT and MRI: a multi-observer study. Int J Radiat Oncol Biol Phys 1999; 43: 57-66. 
50. Merrick GS, Butler WM, Dorsey AT et al. The dependence of prostate postimplant dosimetric quality on $\mathrm{CT}$ volume determination. Int J Radiat Oncol Biol Phys 1999; 44: 1111-1117.

51. Lee WR, deGuzman AF, Bare RL et al. Postimplant analysis of transperineal interstitial permanent prostate brachytherapy: evidence for a learning curve in the first year at a single institution. Int J Radiat Oncol Biol Phys 2000; 46: 83-88.

52. Bice WS, Jr., Prestidge BR, Grimm PD et al. Centralized multiinstitutional postimplant analysis for interstitial prostate brachytherapy. Int J Radiat Oncol Biol Phys 1998; 41: 921-927.

53. Moerland MA, Wijrdeman HK, Beersma R et al. Evaluation of permanent I-125 prostate implants using radiography and magnetic resonance imaging. Int J Radiat Oncol Biol Phys 1997; 37: 927-933.

54. Dubois DF, Prestidge BR, Hotchkiss LA et al. Source localization following permanent transperineal prostate interstitial brachytherapy using magnetic resonance imaging. Int J Radiat Oncol Biol Phys 1997; 39: 1037-1041.

55. Prete JJ, Prestidge BR, Bice WS et al. Comparison of MRI- and CT-based post-implant dosimetric analysis of transperineal interstitial permanent prostate brachytherapy. Radiat Oncol Investig 1998; 6: 90-96.

56. Amdur RJ, Gladstone D, Leopold KA et al. Prostate seed implant quality assessment using MR and CT image fusion. Int J Radiat Oncol Biol Phys 1999; 43: 67-72.

57. Narayana V, Roberson PL, Pu AT et al. Impact of differences in ultrasound and computed tomography volumes on treatment planning of permanent prostate implants. Int J Radiat Oncol Biol Phys 1997; 37: 1181-1185.

58. McLaughlin PW, Narayana V, Drake DG et al. Comparison of MRI pulse sequences in defining prostate volume after permanent implantation. Int J Radiat Oncol Biol Phys 2002; 54: 703-711.

59. Milosevic M, Voruganti S, Blend R et al. Magnetic resonance imaging (MRI) for localization of the prostatic apex: comparison to computed tomography (CT) and urethrography. Radiother Oncol 1998; 47: 277-284.

60. Debois M, Oyen R, Maes F et al. The contribution of magnetic resonance imaging to the three-dimensional treatment planning of localized prostate cancer. Int J Radiat Oncol Biol Phys 1999; 45: 857-865.

61. Polo A, Cattani F, Vavassori A et al. MR and CT image fusion for postimplant analysis in permanent prostate seed implants. Int J Radiat Oncol Biol Phys 2004; 60: 1572-1579.

62. Taussky D, Austen L, Toi A et al. Sequential evaluation of prostate edema after permanent seed prostate brachytherapy using CT-MRI fusion. Int J Radiat Oncol Biol Phys 2005; 62: 974-980.

63. Crook J, McLean M, Yeung I et al. MRI-CT fusion to assess postbrachytherapy prostate volume and the effects of prolonged edema on dosimetry following transperineal interstitial permanent prostate brachytherapy. Brachytherapy 2004; 3: 55-60.

64. Speight JL, Shinohara K, Pickett B et al. Prostate volume change after radioactive seed implantation: possible benefit of improved dose volume histogram with perioperative steroid. Int J Radiat Oncol Biol Phys 2000; 48: 1461-1467.

65. Nag S, Ciezki JP, Cormack R et al. Intraoperative planning and evaluation of permanent prostate brachytherapy: report of the American Brachytherapy Society. Int J Radiat Oncol Biol Phys 2001; 51: 1422-1430.

66. Sahgal A, Roach Mr. Permanent prostate seed brachytherapy: a current perspective on the evolution of the technique and its application. Nat Clin Pract Urol 2007; 4: 658-670.

67. Todor DA, Zaider M, Cohen GN et al. Intraoperative dynamic dosimetry for prostate implants. Phys Med Biol 2003; 48: 1153-1171.
68. Lee EK, Zaider M. Intraoperative dynamic dose optimization in permanent prostate implants. Int J Radiat Oncol Biol Phys 2003; 56: 854-861.

69. Su Y, Davis BJ, Herman MG et al. TRUS-fluoroscopy fusion for intraoperative prostate brachytherapy dosimetry. Stud Health Technol Inform 2006; 119: 532-537.

70. Su Y, Davis BJ, Furutani KM et al. Seed localization and TRUSfluoroscopy fusion for intraoperative prostate brachytherapy dosimetry. Comput Aided Surg 2007; 12: 25-34.

71. French D, Morris J, Keyes M et al. Computing intraoperative dosimetry for prostate brachytherapy using TRUS and fluoroscopy. Acad Radiol 2005; 12: 1262-1272.

72. Gong L, Cho PS, Han BH et al. Ultrasonography and fluoroscopic fusion for prostate brachytherapy dosimetry. Int I Radiat Oncol Biol Phys 2002; 54: 1322-1330.

73. Su Y, Davis BJ, Herman MG et al. Prostate brachytherapy seed localization by analysis of multiple projections: identifying and addressing the seed overlap problem. Med Phys 2004; 31: 12771287.

74. Siewerdsen JH, Moseley DJ, Burch S et al. Volume CT with a flat-panel detector on a mobile, isocentric C-arm: pre-clinical investigation in guidance of minimally invasive surgery. Med Phys 2005; 32: 241-254.

75. Jaffray DA, Siewerdsen JH, Edmundson GK et al. Flat-panel cone-beam CT on a mobile isocentric C-arm for image-guided brachytherapy. Proceedings of SPIE 2002; 4682: 209-217.

76. Siewerdsen JH, Daly MJ, Bachar G et al. Multimode C-arm fluoroscopy, tomosynthesis, and cone-beam CT for imageguided interventions: from proof of principle to patient protocols. Proceedings of SPIE 2007; 6510: 65101A-651011.

77. Westendorp H, Hoekstra CJ, van't Riet A et al. Intraoperative adaptive brachytherapy of iodine- 125 prostate implants guided by C-arm cone-beam computed tomography-based dosimetry. Brachytherapy 2007; 6: 231-237.

78. Grégoire V, Haustermans K, Geets X et al. PET-Based Treatment Planning in Radiotherapy: A New Standard? J Nucl Med 2007; 48: 68-77.

79. Saleem A. Potential of PET in oncology and radiotherapy. Brit J Radiol 2005; Suppl 28: 6-16.

80. Ling CC, Humm J, Larson S et al. Towards multidimensional radiotherapy (MD-CRT): biological imaging and biological conformality. Int J Radiat Oncol Biol Phys 2000; 47: 551-560. 